\title{
Domain-wall branes in Lifshitz theories
}

\author{
Jayne E. Thompson* and Raymond R. Volkas \\ School of Physics, The University of Melbourne, Victoria 3010, Australia
}

(Dated: November 26, 2018)

\begin{abstract}
We analyze whether or not Lifshitz field theories in $4+1$ dimensions may provide ultraviolet-complete domain-wall brane models. We first show that Lifshitz scalar field theory can admit topologically stable domain wall solutions. A Lifshitz fermion field is then added to the toy model, and we demonstrate that 3+1dimensional Kaluza-Klein zero mode solutions do not exist when the four spatial dimensions are treated isotropically. To recover $3+1$-dimensional chiral fermions dynamically localized to the domain wall, we must postulate the breaking of full 4-dimensional rotational symmetry down to the subgroup of rotations which mix the usual 3-dimensional spatial directions and fix the extra-dimensional axis in addition to the anisotropy between space and time.
\end{abstract}

PACS numbers: $11.10 . \mathrm{Kk}, 11.27 .+\mathrm{d}, 11.30 . \mathrm{Cp}$

*Electronic address: j.thompson@pgrad.unimelb.edu.au

†Electronic address: raymondv@unimelb.edu.au 


\section{INTRODUCTION}

Hořava's recent attempt to solve the quantum gravity problem [1, 2] by introducing higher spatial derivative curvature terms, which break Lorentz invariance but ameliorate graviton-loop renormalization effects, has motivated research into other Lorentz violating theories. In particular, there is growing interest in whether the good ultraviolat (UV) behavior of Hořava-Lifshitz gravity may be transferable to certain nonrenormalizable Yang-Mills gauge theories [3], 4]. Models which include higher spatial derivative extensions à la Hořava are collectively referred to as Lifshitz field theories.

The key idea of Lifshitz field theories, in this context, is that by explicitly breaking Lorentz invariance it is possible to write down an action with higher-order spatial derivatives while maintaining quadratic time derivatives and thus the unitarity of the theory. The UV properties of the radiative corrections in the theory are softened by the higher inverse powers of momentum appearing in the propagator. One interesting application is to field-theoretic models featuring extra dimensions of space. It is well-understood that the UV behavior degrades as the number of dimensions is increased. Even increasing the spatial dimensions from three to four causes Yang-Mills theory to be nonrenormalizable. Thus, Lorentz-invariant field-theory models involving extra dimensions are inherently UV incomplete, and must be defined with a UV cutoff. But extra-dimensional Lifshitz theories can be UV complete.

The purpose of this article is to take the standard building blocks for a 4+1-dimensional Lorentz invariant brane world model, collated in [5-7], and see if they can be extended to construct a Lifshitz power counting renormalizable brane world model with quartic leading order spatial derivatives. We are principally interested in whether there is a Lifshitz analogue to the domain-wall brane and if we can dynamically localize fermions. These two elements (together with the DvaliShifman mechanism for dynamical gauge boson localization [8]) form the backbone for a dynamically localized domain-wall brane standard model. Being an entree into the subject, we restrict our analysis here to a toy model featuring just a scalar and a fermion field. A full theory would of course require many more ingredients including gravity and gauge fields, but that is too ambitious a construction to attempt in one step.

We stress that a Lifshitz domain-wall brane model would be markedly different to previous work on extra-dimensional field theories and valuable because preceding work in this field has been entirely on effective field theories and by construction is only predictive up to a UV cutoff 
scale [5, 6].

To this end we take the most general power-counting-renormalizable action for a real Lifshitz scalar field $\phi$ living in 4+1-dimensions which is consistent with a discrete reflection symmetry $\phi \rightarrow-\phi$, where the $Z_{2}$ symmetry is necessary for generating topological boundary conditions. For this model we demonstrate that a kink or domain-wall type of topological defect in the Lifshitz scalar field can condense to form a brane. However when we extend the action to incorporate fermions we find that the standard interpretation of the 3+1-dimensional left-chiral fermion, as the dynamically-localized zero mode in a Kaluza-Klein tower, fails.

In isotropic 4+1-dimensional models, Rubakov and Shaposhnikov [7] argue that at low energies 4+1-dimensional spinors behave like 3+1-dimensional left-chiral fermions localized on the brane. Their justification is: given a solution $\Psi\left(x^{M}\right)$ to the isotropic $4+1$-dimensional Dirac equation, there exists a basis for the space of continuous bounded functions of a single coordinate, $x_{5} \equiv y$, which can be used to project the field $\Psi\left(x^{M}\right)$ onto a 'Kaluza-Klein' tower. This Kaluza-Klein tower contains a normalizable massless $3+1$-dimensional left-chiral zero-mode fermion plus massive $3+1$-dimensional fermions. At low energies only the dynamically-localized massless chiral zero mode in this tower is kinematically allowed and the effective low-energy dynamics carry the right phenomenological signature needed to model the quarks and leptons of the standard model.

We take a solution to the Lifshitz $4+1$-dimensional Dirac equation with quartic leading order spatial derivatives. ${ }^{1}$ We write this solution as a Kaluza-Klein tower of $3+1$-dimensional spinors and we show that this tower does not contain a massless $3+1$-dimensional left-chiral zero mode fermion. We find that spatially isotropic Lifshitz Dirac equations do not have zero mode solutions as part of their Kaluza-Klein towers. To overcome this difficulty we consider other models which do not treat all four spatial dimensions symmetrically. We present the zero mode solution for a model where $4+1$-dimensional Lorentz invariance is broken explicitly to $\mathrm{SO}(3)$ spatial rotational invariance.

In Secs. which we shall be using throughout the rest of this paper. In Sec. IVB of this paper we shall explicitly demonstrate that, for the minimal case of a 4+1-dimensional model with quartic spatial derivatives, the Euler-Lagrange equations for a general power counting renormalizable Lifshitz

\footnotetext{
${ }^{1}$ We choose to stop at quartic order purely for simplicity. A complete theory including renormalizable gravity in $4+1$ dimensions will require an action containing at least order-eight derivatives.
} 
scalar field action have a domain-wall brane solution. In Sec. $\mathrm{V}$ we review dimensional reduction of a fermion action via Kaluza-Klein decomposition and dynamical localization of a candidate 3+1-dimensional fermion. We then explain precisely where this technique fails for Lifshitz extensions. In Sec. VI we discuss alternative models featuring compact extra-dimensions and smaller unbroken spatial symmetries. Our final section is a conclusion.

\section{NOTATION}

We now summarize our notational conventions for the convenience of the reader. The lower case Latin letters will run over spatial coordinates so that $x^{i} \equiv\left(x^{1}, x^{2}, x^{3}, x^{5}\right)$ and can be lowered by contracting with the spatial metric $g_{i j}$. Upper case Latin letters will index 4+1-dimensional space-time coordinates so that $x^{M} \equiv\left(t, x^{i}\right)$. In particular we distinguish the extra-dimensional coordinate with the syntax $x^{5} \equiv y$ and use vector notation for 3-dimensional coordinate vectors, $\vec{x}=\left(x^{1}, x^{2}, x^{3}\right)$. Following standard conventions, the lower case Greek letters $\mu$ and $v$ are reserved for 3+1-dimensional 4 -vectors so that $x^{\mu} \equiv\left(t, x^{1}, x^{2}, x^{3}\right)$. We will apply standard terminology directly to our model without qualifying that we are talking about a 4+1-dimensional, quartic leading order spatial derivatives extension to the standard theory before each statement. We will use these descriptors only when our intention is not clear from the context. The function $\phi\left(x^{M}\right)$ labels the real scalar field which condenses to form the domain-wall brane. The notation $\Psi\left(x^{M}\right)$ refers to the $4+1$-dimensional fermion which we will mode expand in a tower of fields $\psi_{n}\left(x^{\mu}\right)$ corresponding to $3+1$-dimensional massive Dirac particles. We will use the standard symbol $\Delta \equiv g_{i j} \partial^{i} \partial^{j}$. And by $C_{b}(y)$ we mean the space of continuous bounded functions with respect to the $y$-coordinate space.

\section{WEIGHTED SCALING DIMENSIONS}

In a $d+1$-dimensional Lifshitz type theory the space-time manifold is foliated into a product $R \times R^{d}$ with coordinates

$$
\left(t, x^{i}\right)
$$

where $i=1, \ldots, d$. The action is invariant under $d$-dimensional spatial rotations and translations but not under Lorentz boosts. It is useful to introduce the concept of a critical exponent $z$ which characterizes the degree of anisotropy in the space-time manifold. The critical exponent automat- 
ically sets the highest power of $\Delta^{z}=\left(\partial_{i} \partial^{i}\right)^{z}$ appearing in the action for a scalar field, $\phi$, so that if we discard all relevant operators the action possesses a rescaling symmetry,

$$
t \longrightarrow \lambda^{z} t, \quad x^{i} \longrightarrow \lambda x^{i}, \quad \phi \rightarrow \lambda^{\frac{z-d}{2}} \phi .
$$

Because this structure automatically adapts the loop propagators, the correct dimensions to use when evaluating whether the theory is power counting renormalizable are the weighted scaling dimensions in which

$$
[t]_{s}=-z, \quad\left[x^{i}\right]_{s}=-1, \quad[\phi]_{s}=\frac{d-z}{2} .
$$

Hereinafter by $[\tau]_{s}$ we mean the weighted scaling dimensions of $\tau$ which are not to be confused with the usual mass dimensions. Lorentz invariance corresponds to $z=1$.

To make expressions more compact we shall write $d+1_{z}$-dimensions for a foliated space-time manifold with weighted scaling characterized by (3) and in the isotropic space-time case we will drop the ' 1 ' subscript.

Ongoing research is critically examining whether coupling constant running can plausibly cause Lorentz symmetry to emerge as an accidental symmetry in the infrared limit of $z \neq 1$ Lifshitz models [3, 4, 9-12]. The recent insights in Ref. [2] now make it more plausible that the infrared limit of a suitably-defined version of Hořava-Lifshitz gravity can closely resemble pure general relativity, so we can hope that some similar progress will be made for emergent relativity in general. For the purposes of this paper we shall adopt the optimistic stance, though we recognize that the emergence of Lorentz invariance in the infrared without fine-tuning is not a given.

\section{DOMAIN-WALL BRANE}

\section{A. The existence of a domain-wall Brane}

We start by defining a domain-wall brane. It is a kink-like topological defect which defines a (finite thickness) 3+1-dimensional hyperplane to be identified with our universe. The topological defect is provided by a real scalar field with an action $S_{\phi}$ such that the lowest energy density solution to the classical equations of motion, which asymptotically approaches distinct vacua, related by a discrete reflection symmetry, at opposite extremities of the extra-dimension, is a solitary 
wave.

In this section we isolate the real scalar field terms in the action. ${ }^{2}$ We work under the assumption that there is an equivalence relation on the space of all actions such that actions $S_{1}$ and $S_{2}$ are identified if they contain the same terms up to global boundary terms. By consistently following this principle we will discard all terms in the equations of motion which arise from boundary effects. Therefore from an empirical perspective actions belonging to the same equivalence class will be phenomenologically indistinguishable.

To create a domain-wall brane our action must exhibit a discrete reflection symmetry $\phi\left(x^{M}\right) \rightarrow$ $-\phi\left(x^{M}\right)$. To simplify the model we choose to work in flat space-time. ${ }^{3}$ We consider the most general action for $\mathrm{R} \times \mathrm{R}^{4}$ with critical exponent $z=2$ which is consistent with the above and in the methodology of weighted scaling dimensions is renormalizable with a full complement of relevant and marginal operators:

$$
\begin{aligned}
S_{\phi} & =S_{\phi \text { free }}+S_{\phi \text { int }} \\
& =\int d^{5} x\left[\left(\frac{1}{2}\left(\partial_{t} \phi\right)^{2}-\frac{a^{2}}{2 \Lambda^{2}}(\Delta \phi)^{2}-\frac{c^{2}}{2}\left(\partial_{i} \phi\right)^{2}\right)+\left(-\frac{g_{2}}{2} \phi^{2}-\frac{g_{4}}{4 ! \Lambda} \phi^{4}-\frac{b}{4 \Lambda^{3}}\left(\phi \partial_{i} \phi\right)^{2}-\frac{g_{6}}{6 ! \Lambda^{4}} \phi^{6}\right)\right]
\end{aligned}
$$

where $\Lambda$ is being used to keep track of the natural mass dimensions in isotropic space-time. We have absorbed the coupling constant in front of $\left(\partial_{t} \phi\right)^{2}$ into a rescaling of the field $\phi\left(x^{M}\right)$. In the

\footnotetext{
${ }^{2}$ It is physically justifiable to solve the scalar field dynamical equations, and then work out the motion of fermions propagating in the scalar field background because in the framework of domain-wall brane models the fermions are incorporated as a perturbative mode expansion about an empty vacuum state.

${ }^{3}$ Incorporating gravitation will be far from trivial, but not hopeless. First, one would have to face the difficulties [13] that have been identified in the original versions of 3+1-dimensional Hořava gravity [1]. Reference [2] provides a way forward. One would then need to develop a 4+1-dimensional extension, which will require going to a $z=4$ theory, and then establish that the brane tension causes the domain-wall to be a preferred observation hyperplane from which gravity appears to be 3+1-dimensional, and that the effective gravity theory on the wall is sufficiently close to general relativity. For domain-wall branes with 4+1-dimensional general relativity, the answer is provided by the Randall-Sundrum [14, 15] warped metric solution which involves pasting together two semi-infinite regions of anti-de Sitter space-time with a matching junction condition at the location of the brane. This configuration can be used to dynamically localize a 3+1-dimensional Kaluza-Klein zero mode graviton. Something resembling this solution would presumably also have to exist within the putative 4+1-dimensional Hořava gravity theory. The most straight-forward approach would be to verify anti-de Sitter space-time solutions in [2] could be extended to the putative 4+1-dimensional Hořava gravity theory before looking for an analogue to the Randall-Sundrum warped metric ansatz. These challenging problems are well beyond the scope of this paper.
} 
weighted scaling dimensions of our theory we have

$$
[t]_{s}=-z=-2, \quad\left[x^{i}\right]_{s}=-1, \quad[\phi]_{s}=\frac{d-z}{2}=1 .
$$

If we set $[\Lambda]_{s}=0$ then the coupling constants have weighted scaling dimensions:

$$
\begin{array}{rrr}
{\left[a^{2}\right]_{s}=2 z-4=0,} & {\left[c^{2}\right]_{s}=2 z-2=2,} \\
{[b]_{s}=3 z-d-2=0,} & {\left[g_{n}\right]_{s}=d+z-\frac{n(d-z)}{2}=6-n .}
\end{array}
$$

From the above we confirm that $4+1_{2}$-dimensional Lifshitz scalar field theory with a $\phi^{6}$ leading order potential is power counting renormalizable. Note that the parameter $c$ which plays the role of the maximum obtainable velocity in the free particle IR dispersion relation, becomes a running parameter in the quantized theory. It would thus be misleading to absorb the energy-scale-dependent $c^{2}$ by a relative rescaling of spatial and temporal coordinates.

The Klein-Gordon equation obtained from this action using the principle of stationary action is

$$
\partial_{t}^{2} \phi+\frac{a^{2}}{\Lambda^{2}} \Delta^{2} \phi-c^{2} \Delta \phi-\frac{b}{2 \Lambda^{3}} \phi^{2} \Delta \phi-\frac{b}{2 \Lambda^{3}} \phi \partial_{i} \phi \partial^{i} \phi+\frac{g_{6}}{5 ! \Lambda^{4}} \phi^{5}+\frac{g_{4}}{3 ! \Lambda} \phi^{3}+g_{2} \phi=0 .
$$

The domain-wall is the static solitary wave solution to the Klein-Gordon equation which is isotropic in three spatial directions and interpolates between distinct minima of the potential as a function of $y$.

Numerical domain-wall brane solutions to the Klein-Gordon equation have been found for a wide range of parameters. In Fig. 1 we give an example of a numerical domain-wall brane configuration. In addition we can give an explicit example of an analytic domain-wall brane,

$$
\phi=v \tanh (u y),
$$

which is a solution to the Klein-Gordon equation for:

$$
\begin{gathered}
v=\sqrt{-\frac{5 g_{4} \Lambda^{3}}{g_{6}}+\frac{\sqrt{5} \sqrt{-6 g_{6} g_{2}+5 g_{4}^{2} \Lambda^{6}}}{g_{6}}}, \quad u=\sqrt{\frac{b v^{2}}{32 a^{2} \Lambda}-\frac{\sqrt{45 b^{2} v^{4} \Lambda^{2}-16 a^{2} g_{6}^{4} \Lambda^{2}}}{96 \sqrt{5} a^{2} \Lambda^{2}}}, \\
\text { and } g_{6} v^{4}+10 \Lambda\left(-6 b u^{2} v^{2}+\Lambda\left(48 a^{2} u^{4}+\Lambda\left(v^{2} g_{4}-12 c^{2} u^{2} \Lambda\right)\right)\right)=0 .
\end{gathered}
$$




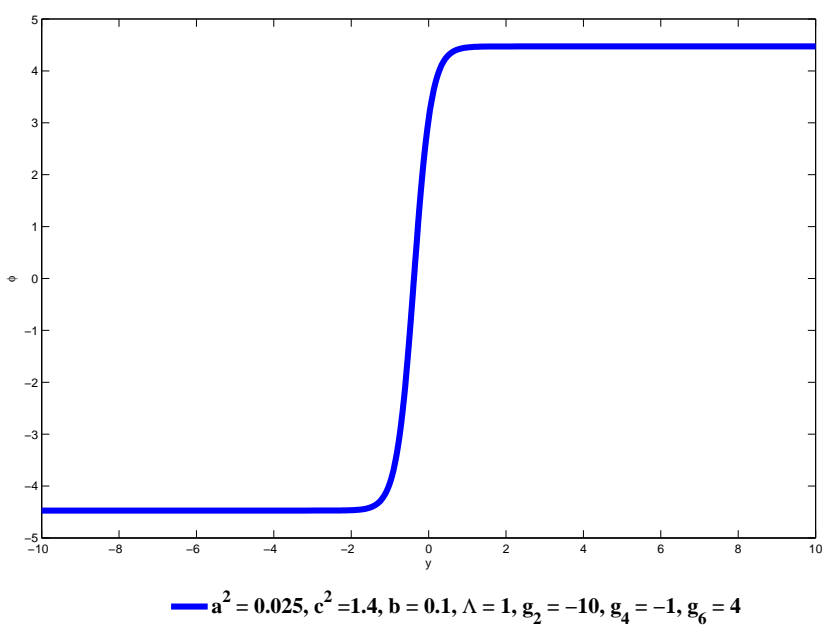

FIG. 1: The graph displays a numerical domain-wall brane solution to the Lifshitz Klein-Gordon equation (7) for a choice of parameters which do not belong to the slice defined in (9).

We clarify that this is not a fine-tuning condition, it is merely a prototypical example of the kink solutions that exist for a large region of parameter space. The numerical solution depicted in Fig. 1 corresponds to a point which is not on this slice. Because we want the solutions described by (8) and (9) to be real valued and because $v$ must be a minimum of the potential for (8) to be a solitary wave, we can bound the allowed region of parameter space by the inequalities $\Lambda, g_{6},-g_{2},-g_{4}>$ 0 . Indeed none of the results presented in this paper are contingent on providentially choosing parameters which satisfy Eq. (97).

This establishes the existence of a domain-wall brane for $R \times R^{4}$ space-time with anisotropic scaling characterized by critical exponent $z=2$.

\section{B. Stability of the domain-wall Brane}

The Lifshitz domain wall is topologically stable for the same reason the usual domain wall is stable: the enforced discrete symmetry, when spontaneously broken, produces disconnected vacua which serve as the boundary conditions for the domain wall solution. The kink is a mapping from the boundary of the real line, parameterizing the extra dimension, onto the disconnected manifold $\{-v, v\}$. This mapping is homotopically non-trivial. Thus, the domain wall is prevented from decaying to the lower-energy spatially-homogeneous vacua $\phi\left(x^{M}\right)= \pm v$. Intuitively, maintaining finite energy density forces the solution to asymptotically approach minima of the potential. Once 
we have fixed $\lim _{y \rightarrow \infty} \phi(y)=v$, and $\lim _{y \rightarrow-\infty} \phi(y)=-v$ there is no way to continuously deform the solution while passing through intermediary states which also have boundary values pinned either at $+v$ or $-v$ and arrive at a solution for which $\lim _{y \rightarrow \pm \infty} \phi(y)=v$ or $\lim _{y \rightarrow \pm \infty} \phi(y)=-v$.

We have not analytically shown that our kink domain-wall brane is the lowest energy solution to the Euler Lagrange equations satisfying these boundary conditions.

In the following discussion we guarantee the kink solution has finite 3+1-dimensional energy density (energy per unit of 3-dimensional volume). Furthermore we show that the kink is stable under perturbations corresponding to a contraction or dilation of the transverse wall width. This suggests the kink is stable because perturbations which alter the profile in any of the $\vec{x}$-coordinate directions are forbidden by $\mathrm{SO}(3)$-rotational invariance. Our background kink solution is invariant under 3-dimensional spatial rotations and there is no preferred direction in the hyperplane orthogonal to the $y$-coordinate, so it does not make sense to say a perturbation has formed along a specific direction in the $\vec{x}$-coordinate space because all directions are relative to an arbitrary choice of reference axis [16].

We are left with conjecturing about perturbations which do not deform the kink in any direction perpendicular to the bulk coordinate axis and do not correspond to rescaling the width of the domain wall. This means that we can only have perturbations which locally deform the kink according to some nonlinear dependence on $y$. These are allowed because translational invariance is spontaneously broken by the condensation of the kink. A full perturbative stability analysis is beyond the scope of this paper.

Our analytic kink (8) has finite 3+1-dimensional energy density given by

$$
\sigma=\frac{v^{2}\left(-23 g_{6} v^{4}+120 \Lambda^{2}\left(24 a^{2} u^{4}+\Lambda\left(v^{2}\left(3 b u^{2}-5 g_{4}\right)-45 g_{2} \Lambda+30 c^{2} u^{2} \Lambda\right)\right)\right)}{5400 u \Lambda^{4}}<\infty
$$

Naturally if we integrate this quantity with respect to the 3-dimensional volume element to obtain the total energy the answer will be infinite. This is the statement that domain-wall branes are technically only solitons in 1+1-dimensions.

In fact in isotropic 4+1-dimensional theories Derrick's theorem [17] implies that all static nonhomogeneous ${ }^{4}$ solutions to the Klein-Gordon equation have infinite total energy, independent of

${ }^{4}$ It should be qualified that the flat vacuum solutions $\phi= \pm v$ which have trivial $x^{i}$-dependence evade Derrick's theorem. 
the precise set of self interaction terms in the action. Intuitively Derrick's theorem arises because a hypothetical static, stable, finite energy density solution, $\phi_{0}\left(x^{i}\right)$, to the Euler Lagrange equations would locally minimize the energy functional. However if we contract the soliton slightly, corresponding to $\phi_{0}\left(x^{i}\right) \rightarrow \phi_{0}\left(k x^{i}\right)$ where $k \approx 1$, then we find the energy functional scales according to

$$
E\left[\phi_{0}\left(k x^{i}\right)\right]=\frac{2 k^{2}-1}{k^{4}} E\left[\phi_{0}\left(x^{i}\right)\right]
$$

and will decrease as $k$ increases. This contradicts the hypothesis that $E\left[\phi_{0}\left(x^{i}\right)\right]$ is minimal.

In Lifshitz theories the higher spatial derivatives change the scaling properties of the energy functional. Effectively the higher spatial derivatives stabilize these perturbations and Derrick's theorem can be evaded. This means we cannot rule out finite total energy solution to the KleinGordon equation in Lifshitz theories. However a finite total energy solution cannot be homogeneous on 3+1-dimensional hyperplanes, so if we wish to avoid breaking 3-dimensional spatial rotational invariance then it will have to be radially symmetric and will therefore correspond to a point-like topological defect. This configuration cannot provide a 3+1-dimensional brane. We find the prospect fascinating for other reasons but have delayed a full investigation to future work.

The higher spatial derivatives in Lifshitz theories also modify the virial theorem. For a 1+1dimensional, solitary wave solution $\phi_{0}(y)$ to the Klein-Gordon equation (7), there is a relation between the contribution to the energy density from the gradient of the field, and the potential energy density. It can be derived from the total 3+1-dimensional energy density

$$
\begin{array}{r}
\sigma\left[\phi_{0}(y)\right]=\int d y[\underbrace{\frac{a^{2}}{2 \Lambda^{2}}\left(\partial_{y}^{2} \phi_{0}\right)^{2}}_{S_{1}}+\underbrace{\frac{c^{2}}{2}\left(\partial_{y} \phi_{0}\right)^{2}}_{S_{2}}+\underbrace{\frac{b}{4 \Lambda^{3}}\left(\phi_{0} \partial_{y} \phi_{0}\right)^{2}}_{S_{3}} \\
+\underbrace{\left[\frac{g_{2}}{2} \phi_{0}^{2}+\frac{g_{4}}{4 ! \Lambda} \phi_{0}^{4}+\frac{g_{6}}{6 ! \Lambda^{4}} \phi_{0}^{6}\right]}_{S_{4}}]=S_{1}+S_{2}+S_{3}+S_{4},
\end{array}
$$

by rescaling the solution $\phi_{0}(y) \rightarrow \phi_{0}(k y)$ and computing $\left.\frac{\partial \sigma\left[\phi_{0}(k y)\right]}{\partial k}\right|_{k=1}=0$. This result is a direct consequence of requiring any static solitary solution $\phi_{0}(y)$ to extremize the energy density. Here the virial relation is:

$$
3 S_{1}+S_{2}+S_{3}=S_{4}
$$


Using this information in the second derivative $\left.\frac{\partial^{2} \sigma\left[\phi_{0}(k y)\right]}{\partial k}\right|_{k=1}$ we find that

$$
\left.\frac{\partial^{2} \sigma\left[\phi_{0}(k y)\right]}{\partial k}\right|_{k=1}=18 S_{1}+2 S_{2}+2 S_{3} .
$$

Provided $b>0$ the integrands of $S_{1}, S_{2}$ and $S_{3}$ are all perfect squares, and we recognize that this quantity must be strictly positive. By corollary a 1+1-dimensional, solitary wave solution $\phi_{0}(y)$ to the Klein-Gordon equation (7) is stable under perturbations corresponding to a contraction or dilation of the transverse wall width.

\section{FERMIONS}

We begin by carefully reviewing why Lorentz-invariant domain-wall branes dynamically localize a massless chiral 3+1-dimensional fermion, and then explain why this phenomenon is impossible in a Lifshitz theory with complete spatial isotropy.

In Lorentz-invariant 4+1-dimensional brane-world models the 4+1-dimensional Dirac equation is

$$
\left[i \Gamma^{M} \partial_{M}-g \phi(y)\right] \Psi\left(x^{N}\right)=0
$$

where $\Gamma^{M}=\left(\gamma^{\mu},-i \gamma^{5}\right)$. A solution to Eq. (15) also satisfies the Klein-Gordon equation

$$
\left(\partial_{t}^{2}-\overrightarrow{\nabla^{2}}-\partial_{y}^{2}+g^{2} \phi(y)^{2}+\gamma^{5} g\left(\partial_{y} \phi(y)\right)\right) \Psi\left(x^{M}\right)=0 .
$$

There exist complete bases $\left\{f_{n L / R}(y)\right\}$ for $C_{b}(y)$ which can be used to project the solution $\Psi\left(x^{N}\right)$ to (15) and (16) onto towers of 3+1-dimensional chiral spinors $\left\{\psi_{n L / R}\left(x^{\mu}\right)\right\}$

$$
\begin{aligned}
\Psi_{i}(x, y) & =\Psi_{0 L}\left(x^{M}\right)+\sum_{n>0}\left\{\Psi_{n L}\left(x^{M}\right)+\Psi_{n R}\left(x^{M}\right)\right\}, \\
& =f_{0 L}(y) \psi_{0 L}\left(x^{\mu}\right)+\sum_{n>0}\left\{f_{n L}(y) \psi_{n L}\left(x^{\mu}\right)+f_{n R}(y) \psi_{n R}\left(x^{\mu}\right)\right\},
\end{aligned}
$$

where we have introduced the notation $\Psi_{n L / R}\left(x^{M}\right)=f_{n L / R}(y) \psi_{n L / R}\left(x^{\mu}\right)$. We will also write $\Psi_{n}\left(x^{M}\right)=$ $\Psi_{n L}\left(x^{M}\right)+\Psi_{n R}\left(x^{M}\right)$. There are as many decompositions of this type as there are complete sets of continuous bounded functions of $y$. But, for two reasons, there is one basis that is special. First, for this basis the $\Psi_{n}\left(x^{M}\right)$ appearing in (17) are independent solutions to the Dirac equation (15) 
which satisfy orthonormality conditions in a rigged Hilbert space. Second, each 3+1-dimensional spinor in this tower satisfies the 3+1-dimensional Dirac equation for a particle with mass $m_{n}$ :

$$
\begin{aligned}
i \gamma^{\mu} \partial_{\mu} \psi_{n L}\left(x^{\mu}\right) & =m_{n} \psi_{n R}\left(x^{\mu}\right), \\
i \gamma^{\mu} \partial_{\mu} \psi_{n R}\left(x^{\mu}\right) & =m_{n} \psi_{n L}\left(x^{\mu}\right) .
\end{aligned}
$$

The 3+1-dimensional left-chiral zero-mode $\psi_{0 L}\left(x^{\mu}\right)$ spinor satisfies (18) with $m_{0}=0$. Equation (17) does not contain a right-handed massless 3+1-dimensional spinor. Directly calculating the form of $f_{0 R}(y)$ when $\psi_{0 R}\left(x^{\mu}\right)$ is a massless $3+1$-dimensional right-chiral fermion and $f_{0 R}(y) \psi_{0 R}\left(x^{\mu}\right)$ is a solution to (15) reveals that $f_{0 R}(y)$ does not belong to $C_{b}(y)$ and hence can not form part of a basis for this space.

From Eq. (18) it follows that each $\psi_{n L}\left(x^{\mu}\right)+\psi_{n R}\left(x^{\mu}\right)$ satisfies a $3+1$-dimensional Klein-Gordon equation

$$
\begin{aligned}
& \left(\partial_{t}^{2}-\vec{\nabla}^{2}\right) \psi_{n L}\left(x^{\mu}\right)=m_{n}^{2} \psi_{n L}\left(x^{\mu}\right) \\
& \left(\partial_{t}^{2}-\vec{\nabla}^{2}\right) \psi_{n R}\left(x^{\mu}\right)=m_{n}^{2} \psi_{n R}\left(x^{\mu}\right) .
\end{aligned}
$$

If we use unbroken 3+1-dimensional Poincaré invariance to expand $\psi_{n L}\left(x^{\mu}\right)+\psi_{n R}\left(x^{\mu}\right)$ in terms of plane waves then we find that the dispersion relation describing the propagation of the $3+1$ dimensional spinor $\psi_{n L}\left(x^{\mu}\right)+\psi_{n R}\left(x^{\mu}\right)$ with energy-momentum 4-vector $p_{n}=\left(\omega_{n}, \vec{p}_{n}\right)$ is

$$
\omega_{n}^{2}-\vec{p}_{n} \cdot \vec{p}_{n}=m_{n}^{2}
$$

From the perspective of a 3+1-dimensional observer these particles can now be given the interpretation of propagating free particles with masses $m_{n}$ which transform according to a spin- $1 / 2$ representation of an embedded 3+1-dimensional Lorentz space-time symmetry. For each mode $\Psi_{n}\left(x^{M}\right)$ which appears in (17) a 3+1-dimensional observer will see a resonance in the detector at energy $m_{n}$ caused by what he perceives to be a massive fermion $\psi_{n L}\left(x^{\mu}\right)+\psi_{n R}\left(x^{\mu}\right)$. At low energies only the left-chiral zero-mode $\psi_{0 L}\left(x^{\mu}\right)$ will be detectable.

We choose to work with this special basis because we have a physical interpretation for the individual modes $\Psi_{n}\left(x^{M}\right)$. This interpretation allows us to argue that at low energies there is a candidate 3+1-dimensional massless chiral fermion and explain why parity is broken (the kink 
localizes a zero mode of one chirality only).

We find this special basis and the allowed masses, $m_{n}$, of the $3+1$-dimensional spinors present in (15) by solving the eigenvalue problem:

$$
\left[-\partial_{y}^{2}+g^{2} \phi(y)^{2} \pm g \partial_{y} \phi(y)\right] f_{n L / R}(y)=m_{n}^{2} f_{n, L / R}(y) .
$$

Sturm-Liouville theory determines the existence and completeness of eigensystems generated by (21).

We can check these conditions are consistent and that the eigenfunctions in (21) are the correct 'special basis' functions $\left\{f_{n L / R}(y)\right\}$ to use in (15). To do this we substitute $\Psi_{n}\left(x^{M}\right)$ into the 4+1dimensional Dirac equation and use (18) to simplify. After isolating the coefficients of the 3+1dimensional spinors $\psi_{n L}\left(x^{\mu}\right)$ and $\psi_{n R}\left(x^{\mu}\right)$, which correspond to independent degrees of freedom, and setting each coefficient equal to zero independently we arrive at:

$$
\begin{aligned}
{\left[-\partial_{y}-g \phi(y)\right] f_{n L}(y) } & =m_{n} f_{n R}(y), \\
{\left[\partial_{y}-g \phi(y)\right] f_{n R}(y) } & =m_{n} f_{n L}(y) .
\end{aligned}
$$

Uncoupling this first order system automatically generates the two second order differential equations in (21). We can also show that each mode $\Psi_{n}\left(x^{M}\right)$ satisfies the Klein-Gordon equation (16) by using separation of variables and Eqs. (21) and (19).

We need to look at how the situation changes in the $4+1_{2}$-dimensional case.

First we must clarify what we mean by a fermion in $4+1_{2}$-dimensional space-time. We consider $\Psi\left(x^{M}\right)$ to be a spinorial wave function on $R \times R^{4}$ which transforms under $\mathrm{SO}(4)$ spatial rotations $x^{j} \rightarrow x^{j}=O_{k}^{j} x^{k} \approx\left(\delta_{k}^{j}-\epsilon_{k i}^{j} \theta^{i}\right) x^{k}$ according to

$$
\Psi\left(x^{M}\right) \longrightarrow S(O) \Psi\left(x^{M}\right), \quad S(O)=e^{\omega^{j k} \Sigma_{j k}}
$$

where for convenience $\omega^{j k}=\epsilon^{j k i} \theta_{i}$ and $\Sigma_{j k}=-\frac{i}{4}\left[\Gamma_{j}, \Gamma_{k}\right]$. As we are talking about 4-dimensional spatial rotations here the $\Gamma^{j} \in\left\{\gamma^{1}, \gamma^{2}, \gamma^{3}, \gamma^{5}\right\}$.

In the IR limit we are assuming the theory exhibits an accidental Lorentz symmetry. This Lorentz group incorporates a 3+1-dimensional subgroup given by the subset of $\mathrm{SO}(3)$ spatial rotations augmented by boosts in the $3+1$-dimensional coordinate space which are a symmetry of the low-energy Lagrangian. Chirality will be restored in this low-energy Lorentz-invariant theory 
provided we can localize a Kaluza-Klein zero-mode fermion and provided this zero-mode fermion is an eigenstate of the $\gamma^{5}$ operator which commutes with the 3+1-dimensional Lorentz subgroup.

We interpret the $4+1_{2}$-dimensional Dirac equation, for $\Psi\left(x^{M}\right)$, to be an $\mathrm{SO}(4)$ covariant equation which will putatively flow towards (15) in the low energy limit. Therefore the $4+1_{2}$ dimensional Dirac equation will contain all the operators present in (15). To suppress perturbative fermion loop diagram contributions to transition amplitudes we must incorporate second order spatial derivatives. We choose to do this in such a way that the propagation of the free $4+1_{2}$ dimensional scalar boson and free $4+1_{2}$-dimensional fermion will be described by the same dispersion relation. This means a solution to the free field $4+1_{2}$-dimensional Dirac equation also satisfies a Klein-Gordon equation which contains the same differential operators as the KleinGordon equation for the free scalar field. We will absorb the coefficient in front of the temporal derivative into a rescaling of the field $\Psi\left(x^{M}\right)$. Therefore the minimal structure necessary for a viable Dirac equation is:

$$
\left[i \Gamma^{0} \partial_{t}+\beta i \Gamma^{i} \partial_{i}-\alpha \Delta-g \phi(y)\right] \Psi\left(x^{M}\right)=0
$$

The associated Klein-Gordon equation is

$$
0=\left[\partial_{t}^{2}-\beta^{2} \Delta+(\alpha \Delta+g \phi(y))^{2}-g \beta \gamma^{5}\left(\partial_{y} \phi(y)\right)\right] \Psi\left(x^{M}\right) .
$$

Comparing the above equation to (7) will confirm that the free field kinetic operators are the same.

We will argue that for our $z=2$ model, the Kaluza-Klein 'zero mode' $f_{0 L}(y) \psi_{0 L}\left(x^{\mu}\right)$ is not a solution to the $4+1_{2}$-dimensional Dirac equation. It is therefore not present in the Kaluza-Klein tower expression (17) and can not provide a candidate $3+1_{2}$-dimensional left-chiral fermion.

We follow the logic: If $f_{0 L}(y) \psi_{0 L}\left(x^{\mu}\right)$ is a solution to the $4+1_{2}$-dimensional Dirac equation then it is also a solution of the Klein-Gordon equation (25). Therefore by taking the contrapositive of this statement: if $f_{0 L}(y) \psi_{0 L}\left(x^{\mu}\right)$ is not a solution to (25) then it is not a solution to the $4+1_{2^{-}}$ dimensional Dirac equation. We show $f_{0 L}(y) \psi_{0 L}\left(x^{\mu}\right)$ is not a solution to (25).

We do this because the operator in the Klein-Gordon equation (25) is diagonal when acting on a Kaluza-Klein tower (17) and for the left-chiral zero mode this operator can be simplified to an identity matrix acting on the spinor space multiplied by a differential operator. For the left-chiral 
zero mode Eq. (25) is

$$
0=\left[\partial_{t}^{2}-\beta^{2} \Delta+(\alpha \Delta+g \phi(y))^{2}+g \beta\left(\partial_{y} \phi(y)\right)\right] f_{0 L}(y) \psi_{0 L}\left(x^{\mu}\right)
$$

It should immediately become clear that the kinetic operator in (26) contains terms like $2 \alpha \partial_{y}^{2} \vec{\nabla}^{2}$ which act on both the $f_{0 L}(y)$ component of this solution and the $\psi_{0 L}\left(x^{\mu}\right)$ component of the solution. This is different from the isotropic 4+1-dimensional Klein-Gordon equation (16). It means that using separation of variables in Eq. (26) will no longer work.

For any solution $f_{0 L}(y) \psi_{0 L}\left(x^{\mu}\right)$ to Eq. (26), we can use unbroken $3+1_{2}$-dimensional Poincaré invariance to expand $\psi_{0 L}\left(x^{\mu}\right)$ in terms of plane waves

$$
\psi_{0 L}\left(x^{\mu}\right)=\sum d^{4} p_{0} e^{-i\left(\omega_{0} t-\overrightarrow{p_{0}} \cdot \vec{x}\right)} \psi_{0 L}\left(p_{0}^{\mu}\right)
$$

where the coefficient

$$
\psi_{0 L}\left(p_{0}^{\mu}\right)=\frac{1}{(2 \pi)^{4}} \int d^{4} x e^{i\left(\omega_{0} t-i \overrightarrow{p_{0}} \cdot \vec{x}\right)} \psi_{0 L}\left(x^{\mu}\right)
$$

and the allowed values of $p_{0}^{\mu}$ are determined by substituting this expansion into (26). At this stage, before finding the allowed values of $p_{0}^{\mu}$, we must be clear that $p_{0}^{\mu}$ can not be a function of $y$.

First, if $p_{0}^{\mu}$ depends on $y$ then Eq. (27) will imply that $\psi_{0 L}\left(x^{\mu}\right)$ depends on $y$ and our interpretation of the Kaluza-Klein tower (17) as a projection of the solution $\Psi\left(x^{M}\right)$ to the $4+1_{2}$-dimensional Dirac equation down onto a space of $3+1_{2}$-dimensional spinors $\psi_{n L / R}\left(x^{\mu}\right)$ using a complete basis for $C_{b}(y)$ is incorrect. This will mean there is a logical inconsistency in our theory.

Second, there will be phenomenological problems for our domain-wall brane model because when the brane is created by a soliton it has finite width. For the analytic solution given in (8) this width is characterized by $1 / u$. If $p_{0}^{\mu}$ depends on $y$ then a particle which starts on the $y<0$ side of the brane and propagates minutely in the $y$-direction to the $y>0$ side will experience a sudden change in energy or momentum $\vec{p}_{0}$. This is particularly true if $p_{0}^{\mu}$ depends in any way on the derivative $\partial_{y} \phi(y)$ of the topological defect which is large around $y=0$ because $\phi(y)$ is varying rapidly at the location of the wall. Shrinking the width of the wall will make the gradient of $\phi(y)$ larger around $y=0$ and make any dependence of $p_{0}^{\mu}$ on precise $y$-coordinate location more pronounced. With this assumption we derive the dispersion relations: 


$$
\begin{array}{r}
0=\left[\omega_{0}^{2}-\alpha^{2}\left(\vec{p}_{0}^{2}\right)^{2}\right] f_{0 L}(y)+\vec{p}_{0}^{2} \underbrace{\left[2 \alpha^{2} \partial_{y}^{2}+2 \alpha g \phi(y)-\beta^{2}\right]}_{o 1} f_{0 L}(y) \\
-\underbrace{\left[\left(\alpha \partial_{y}^{2}+g \phi(y)\right)^{2}-\beta^{2} \partial_{y}^{2}+g \beta\left(\partial_{y} \phi(y)\right)\right]}_{o 2} f_{0 L}(y) .
\end{array}
$$

For this dispersion relation to be $y$-independent $f_{0 L}(y)$ will have to be an eigenfunction of both $o 1$ and $o 2$. Although we are free to choose any complete basis we like, there is no function $f_{0 L}(y)$ which is a simultaneous eigenfunction of both the operators $o 1$ and $o 2$. Thus we have a contradiction. There is no 'zero mode' solution to the $4+1_{2}$-dimensional Dirac equation and hence no candidate $3+1_{2}$-dimensional left-chiral fermion at any energy regime.

We follow the source of this problem back to the higher spatial derivative appearing among the kinetic terms in the Klein-Gordon equation. These higher spatial derivative operators include terms like $2 \alpha^{2} \partial_{y}^{2} \vec{\nabla}^{2}$ which acted on both $f_{0 L}(y)$ and $\psi_{0 L}\left(x^{\mu}\right)$ and ultimately caused the operator $o 1$ to appear in Eq. (29). Since the problem is due to the kinetic operator in the Klein-Gordon equation (25) it cannot be fixed by adding additional interaction terms to (24). These higher spatial derivative terms will be present in any $4+1_{z}$-dimensional $\mathrm{SO}(4)$ invariant model with $z \geq 2$. They are uniquely fixed by $\mathrm{SO}(4)$ spatial rotational invariance, so the culprit in this theory is spatial dimensional democracy.

\section{BREAKING SPATIAL ISOTROPY}

If we intend to project out effective $3+1_{2}$-dimensional left-chiral fermions via Kaluza-Klein decomposition then we can not treat all four spatial dimensions symmetrically. This immediately prompts us to consider the alternative compact extra-dimensions paradigm.

Consider a model where space-time is a direct-product of a 3+1-dimensional manifold, $\mathcal{M}^{4}$, and a compact extra-dimension with an orbifolding symmetry (the extra-dimension is identified into cosets $\left.S^{1} / Z_{2}\right)$. Equip the space $\mathcal{M}^{4} \times S_{1} / Z_{2}$ with a preferred foliation into constant time sheets and impose anisotropic scaling characterized by critical exponent $z=2$.

As before the standard tactic for recovering 3+1-dimensional chiral fermions is to KaluzaKlein mode expand $\Psi\left(x^{\mu}, y\right)$ using a complete set $\left\{f_{n L / R}(y)\right\}$ of eigenfunctions of the $y$-dependent component of the Dirac equation. The crucial difference is that the boundary conditions are fixed 
by periodicity with respect to $y$ of the wave-function and the transformation of the spinor under the orbifolding symmetry. In the isotropic space-time case these boundary conditions eliminate the right-handed zero mode profile function and 3+1-dimensional chirality discriminating physics is again an emergent low energy phenomenon. Unfortunately the conceptual differences between compact and infinite extra-dimensions change the boundary conditions for the Euler-Lagrange equations rather than the form of the kinetic operator. Because compact extra-dimension models still rely on the existence of a separable solution $f_{0 L}(y) \psi_{0 L}\left(x^{\mu}\right)$ to the Klein-Gordon equation (25) they will strike the same problems.

The only option left is to strongly break 4+1-dimensional Lorentz invariance to $\mathrm{SO}(3)$ spatial rotational invariance. If we keep our anisotropic scaling with $z=2$ then we can now write our Dirac equation as

$$
\left[i \Gamma^{0} \partial_{t}+i \beta \Gamma^{i} \partial_{i}+\alpha \Gamma^{0} \vec{\nabla}^{2}+\lambda \partial_{y}^{2}-g \phi(y)\right] \Psi\left(x^{M}\right)=0 .
$$

In constructing Eq. (30) we have deliberately multiplied the differential operator $\vec{\nabla}^{2}$ by a matrix which anti-commutes with $\Gamma^{5}$. This matrix can not be $\Gamma^{1}, \Gamma^{2}$ or $\Gamma^{3}$ because we are demanding $\mathrm{SO}$ (3) rotational invariance, therefore it must be $\Gamma^{0}$. We need to introduce this matrix operator for two reasons. First, because it engineers a Klein-Gordon equation which has a separable solution of the form $\Psi_{0 L}\left(x^{M}\right)=\psi_{0 L}\left(x^{\mu}\right) f_{0 L}(y)$. Explicitly the form of the Klein-Gordon equation is

$$
\left[-\left(i \partial_{t}+\alpha \vec{\nabla}^{2}\right)^{2}-\beta^{2} \vec{\nabla}^{2}+\left(\lambda \partial_{y}^{2}-g \phi(y)\right)^{2}-\beta^{2} \partial_{y}^{2}-\beta \gamma^{5}\left(\partial_{y} g \phi(y)\right)\right] \Psi\left(x^{M}\right)=0 .
$$

There are no operators in Eq. (31) which operate on both the $x^{\mu}$ and $y$ coordinate spaces simultaneously.

Second, to enable the effective $3+1_{2}$-dimensional theory to be chiral, we need the left- and rightchiral components of the zero mode to be independent solutions of Eq. (30). We can then arrange for the right-chiral profile function $f_{0 R}(y)$ not to belong to $C_{b}(y)$ so that the right chiral 'zero mode' is excluded from the Kalza-Klein tower. We collect the $x^{\mu}$-coordinate space differential operators in (30) and interpret the $3+1_{2}$-dimensional zero mode massless spinor to be the solution of

$$
\left[i \gamma^{0} \partial_{t}+i \beta\left(\gamma^{1} \partial_{1}+\gamma^{2} \partial_{2}+\gamma^{3} \partial_{3}\right)+\alpha \gamma^{0} \vec{\nabla}^{2}\right] \psi_{0 L / R}\left(x^{\mu}\right)=0
$$

It is easy to check that $\psi_{0 L}\left(x^{\mu}\right)$ and $\psi_{0 R}\left(x^{\mu}\right)$ will only be independent solutions to (32) when the $\vec{\nabla}^{2}$ differential operator term in Eq. (32) is multiplied by $\gamma^{0}$. 
After using (32) to simplify the algebra we find that $\Psi_{0 L / R}\left(x^{M}\right)=f_{0 L / R}(y) \psi_{0 L / R}\left(x^{\mu}\right)$ will be a solution to (30) if

$$
\begin{aligned}
& 0=\lambda \partial_{y}^{2} f_{0 L}(y)-\beta \partial_{y} f_{0 L}(y)-g \phi(y) f_{0 L}(y), \\
& 0=\lambda \partial_{y}^{2} f_{0 R}(y)+\beta \partial_{y} f_{0 R}(y)-g \phi(y) f_{0 R}(y)
\end{aligned}
$$

If we make the substitution $f_{0 L / R}(y)=e^{ \pm \beta y / 2 \lambda} F_{0 L / R}(y)$ then these equations can be converted into a time independent Schrödinger equation for $F_{0 L / R}(y)$ :

$$
\left[-\partial_{y}^{2}+\frac{g}{\lambda} \phi(y)+\frac{\beta^{2}}{4 \lambda^{2}}\right] F_{0 L / R}(y)=0 .
$$

We observe that for the kink form of $\phi(y)$ given in (8) and parameters belonging to the open set $4 g v \lambda>\beta^{2}$, the solution $F_{0 L / R}(y)$ to (34) describes a freely propagating particle in the region $y<u \operatorname{arctanh}\left(-\beta^{2} / 4 g v \lambda\right)$ which is incident on a potential barrier for $y>u \operatorname{arctanh}\left(-\beta^{2} / 4 g v \lambda\right)$. Because all numerical solutions have the same kink like behavior we can extend this analysis to cover all domain-wall branes. We treat the analytic case here because it has a closed form expression for the location of the potential barrier in (34). We can use the WKB method to find an approximate solution to (34) and write the zero mode profile functions in terms of our analytic solution (8) for $\phi(y)$, as

$$
f_{0 L / R}(y) \approx e^{ \pm \beta y / 2 \lambda} \begin{cases}\sqrt[4]{\frac{4 \lambda^{2}}{4 \lambda g \phi(y)+\beta^{2}}} e^{-\int_{u \operatorname{arctanh}\left(-\beta^{2} / 4 g \nu \lambda\right)}^{y} d y^{\prime} \sqrt{g \phi\left(y^{\prime}\right) / \lambda+\beta^{2} / 4 \lambda^{2}}} & : y>u \operatorname{arctanh}\left(-\beta^{2} / 4 g \nu \lambda\right) \\ \sqrt[4]{\frac{4 \lambda^{2}}{-\beta^{2}-4 \lambda g \phi(y)}} e^{i \int_{u \operatorname{arctanh}\left(-\beta^{2} / 4 g \nu \lambda\right)}^{y} d y^{\prime} \sqrt{-\beta^{2} / 4 \lambda^{2}-g \phi\left(y^{\prime}\right) / \lambda}}: y<u \operatorname{arctanh}\left(-\beta^{2} / 4 g \nu \lambda\right) .\end{cases}
$$

This approximation breaks down at the cusp of (35), $y=u \operatorname{arctanh}\left(-\beta^{2} / 4 g v \lambda\right)$. Provided $\beta>0$ the profile function $f_{0 R}(y)$ grows exponentially as $y \rightarrow-\infty$ and the right handed 'zero mode' will not belong to our Kaluza-Klein tower. In addition $f_{0 L}(y)$ satisfies $\lim _{y \rightarrow \pm \infty} f_{0 L}(y)=0$. This follows from substituting the asymptotic form $\lim _{y \rightarrow \pm \infty} \phi(y) \rightarrow \pm g v$ for the analytic kink $\phi(y)$ given in (8) into the expression for $f_{0 L}(y)$ (35) and taking the appropriate limits. The left-chiral profile function $f_{0 L}(y)$ decays exponentially in both directions and therefore given $\epsilon>0, \exists y(\epsilon)$ such that $\left|f_{0 l}(y)\right|<\epsilon, \forall|y| \geq y(\epsilon)$ and on the compact region $|y|<y(\epsilon)$ we can argue that $f_{0 L}(y)$ is continuous and hence attains a finite maximum, thus $f_{0 L}(y)$ is bounded.

We can now solve Eq. (32) to obtain the form of the $3+1_{2}$-dimensional spinor $\psi_{0 L}\left(x^{\mu}\right)$. We 
choose to write the full result for the left-chiral zero mode in terms of the solution for $f_{0 L}(y)$ given in (35) as:

$$
\Psi_{0 L}\left(x^{M}\right)=N f_{0 L}(y)\left(\begin{array}{c}
\chi_{+} \\
0
\end{array}\right) e^{-i\left(\omega_{0} t-\overrightarrow{p_{0}} \cdot \vec{x}\right)}
$$

where $N$ is a normalization constant, the two component column vector $\chi_{+}$is the eigenstate of the helicity operator ${ }^{5}$ corresponding to $\vec{\sigma} \cdot \vec{p}_{0} \chi_{+}=\left|\vec{p}_{0}\right| \chi_{+}$, the lower two components of the four component spinor are zero and the energy and momentum of the plane wave $p_{0}^{\mu}=\left(\omega_{0}, \vec{p}_{0}\right)$ is fixed by the dispersion relation derived by substituting this solution into the Klein-Gordon equation (31) to obtain

$$
\left(\omega_{0}-\alpha \vec{p}_{0}^{2}\right)^{2}-\beta^{2} \vec{p}_{0}^{2}=0
$$

This energy-momentum relation for the $3+1_{2}$-dimensional subspace no longer depends on $y$.

However if we derive the free scalar field Klein-Gordon equation directly from a $z=2, \operatorname{SO}(3)$ invariant action for $\phi\left(x^{M}\right)$ we will arrive at

$$
\left(\partial_{t}^{2}-\frac{a_{1}^{2}}{\Lambda^{2}}\left(\vec{\nabla}^{2}\right)^{2}-\frac{a_{2}^{2}}{\Lambda^{2}} \partial_{y}^{4}-\frac{a_{3}^{2}}{\Lambda^{2}} \partial_{y}^{2} \vec{\nabla}^{2}-c_{1}^{2} \vec{\nabla}^{2}-c_{2}^{2} \partial_{y}^{2}\right) \phi\left(x^{M}\right)=0 .
$$

The domain-wall brane (8) will still be a solution to (38). In fact in the spatially anisotropic scenario where Lorentz invariance is broken directly to $\mathrm{SO}(3)$ spatial rotational invariance and the real scalar field Klein-Gordon equation (involving self interaction terms) has the form:

$$
\begin{aligned}
& \partial_{t}^{2} \phi-\frac{a_{1}^{2}}{\Lambda^{2}}\left(\vec{\nabla}^{2}\right)^{2} \phi-\frac{a_{2}^{2}}{\Lambda^{2}} \partial_{y}^{4} \phi-\frac{a_{3}^{2}}{\Lambda^{2}} \partial_{y}^{2} \vec{\nabla}^{2} \phi-c_{1}^{2} \vec{\nabla}^{2} \phi-c_{2}^{2} \partial_{y}^{2} \phi-\frac{b_{1}}{2 \Lambda^{3}} \phi^{2} \vec{\nabla}^{2} \phi \\
& -\frac{b_{1}}{2 \Lambda^{3}} \phi \vec{\nabla} \phi \cdot \vec{\nabla} \phi-\frac{b_{2}}{2 \Lambda^{3}} \phi^{2} \partial_{y}^{2} \phi-\frac{b_{2}}{2 \Lambda^{3}} \phi \partial_{y} \phi \partial_{y} \phi-\frac{g_{6}}{5 ! \Lambda^{4}} \phi^{5}-\frac{g_{4}}{3 ! \Lambda} \phi^{3}-g_{2} \phi=0,
\end{aligned}
$$

our solitary wave domain-wall brane (8) will be a solution provided the free parameters satisfy (9) with the identifications $a \equiv a_{2}, c \equiv c_{2}$, and $b \equiv b_{2}$.

From a comparison of (38) and (31) we infer that the fermions and bosons will now propagate according to different dispersion relations. It is easy to check that if we insist on writing down a

\footnotetext{
${ }^{5}$ The helicity operator gives the component of the spin of $\psi_{0 L}\left(x^{\mu}\right)$ in the direction of propagation in the 3-dimensional $\vec{x}$ coordinate space. It is defined in terms of a vector of Pauli matrices $\vec{\sigma}=\left(\sigma_{1}, \sigma_{2}, \sigma_{3}\right)$ and the particle's 3momentum, $\vec{p}_{0}$, as $\vec{\sigma} \cdot \vec{p}_{0} /\left|\vec{p}_{0}\right|$.
} 
Dirac equation for which the solutions $\Psi\left(x^{M}\right)$ are also solutions to (38) for the case $a_{3}=0$ (this will circumvent our previous problem of mixed partial derivative operators) then we will have to use a 7-dimensional Clifford algebra. This forces the smallest representation for our spinor $\Psi\left(x^{M}\right)$ to consist of 8-component column matrices and ultimately once this Dirac equation has been solved we find that the 'zero mode' has too many degrees of freedom to be given the interpretation of a massless chiral fermion.

\section{CONCLUSION}

In this paper we have examined a $4+1_{z}$-dimensional Lifshitz scalar field theories, with critical exponent $z=2$. We have demonstrated that a topological defect in the scalar field can spontaneously condense to form a domain-wall brane. We consider the dynamics of a Lifshitz fermion in this background and show that a $3+1_{2}$-dimensional left chiral Kaluza-Klein zero mode fermion will become trapped on the wall only when four fold spatial rotational invariance is strongly broken to three-fold rotational invariance. So it is not possible to keep 4-dimensional spatial isotropy and localize a Kalza-Klein zero mode candidate chiral fermions in 4+1-dimensional Lifshitz domainwall brane models. While we worked with $z=2$ theory for simplicity, we do not expect the results to be qualitatively different for the more realistic case of $z=4$, necessary to have a renormalizable quantum theory of gravity in $4+1$ dimensions. Many challenges remain before a realistic UVcomplete domain-wall brane model could be contemplated, including the dynamical localization of gravity in some type of Hořava-Lifshitz theory, and the incorporation of dynamically-localized gauge fields.

\section{Acknowledgments}

We thank D. P. George and A. Kobakhidze for helpful discussions. In particular we acknowledge D. P. George independently proposed that a Lifshitz domain-wall brane model will have improved UV-behaviour leading to a strengthening of the Dvali-Shifman conjecture for gaugefield localization. This work was supported in part by the Australian Research Council and in part 
by the Puzey Bequest to the University of Melbourne.

[1] P. Hořava, Phys. Rev. D 79, 084008 (2009) [arXiv:0901.3775]; JHEP 03, 020 (2009) [arXiv:0812.4287]; Phys. Rev. Lett. 102, 161301 (2009) [arXiv:0902.3657].

[2] P. Hořava and C. M. Melby-Thompson, arXiv:1007.2410.

[3] R. Iengo, J. G. Russo and M. Serone, JHEP 0911, 020 (2009) [arXiv:0906.3477].

[4] R. Iengo and M. Serone, Phys. Rev. D 81, 125005 (2010) [arXiv:1003.4430].

[5] R. Davies, D. P. George and R. R. Volkas, Phys. Rev. D 77 (2008) 124038 [arXiv:0705.1584].

[6] J. E. Thompson and R. R. Volkas, Phys. Rev. D 80, 125016 (2009) [arXiv:0908.4122].

[7] V. A. Rubakov and M. E. Shaposhnikov, Phys. Lett. B 125 (1983) 136.

[8] G. R. Dvali and M. A. Shifman, Phys. Lett. B 396 (1997) 64 [Erratum-ibid. B 407 (1997) 452] [arXiv:hep-th/9612128].

[9] D. Anselmi, Annals Phys. 324, 874 (2009) [arXiv:0808.3470].

[10] D. Anselmi, Annals Phys. 324, 1058 (2009) [arXiv:0808.3474].

[11] D. Anselmi, Phys. Rev. D 79, 025017 (2009) [arXiv:0808.3475].

[12] B. Chen and Q. G. Huang, Phys. Lett. B 683, 108 (2010) [arXiv:0904.4565].

[13] G. Charmousis et al., JHEP 08, 070 (2009) [arXiv:0905.2579]; M. Li and Y. Pang, JHEP 0908, 015 (2009) [arXiv:0905:2751]; D. Blas, O. Pujolàs and S. Sibiryakov, JHEP 10, 029 (2009) [arXiv:0906.3046]; Phys. Rev. Lett. 104, 181302 (2010) [arXiv:0909.3525]; A. Kobakhidze, arXiv:0906.5401.

[14] L. Randall and R. Sundrum, Phys. Rev. Lett. 83 (1999) 3370 [arXiv:hep-ph/9905221].

[15] L. Randall and R. Sundrum, Phys. Rev. Lett. 83 (1999) 4690 [arXiv:hep-th/9906064].

[16] L. Pogosian and T. Vachaspati, Phys. Rev. D 62, (2000) 123506 [arXiv:hep-ph/0007045].

[17] G H. Derrick, J. Math. Phys. 5, 1252 (1964) 